\title{
Mirror Writing: An Advantage for the Left-Handed?
}

\author{
JYOTSNA VAID \\ Texas A \& M University \\ AND \\ JoAN StILes-Davis \\ University of California, San Diego
}

\begin{abstract}
It has been claimed that left-handed individuals are better than right-handers at writing in a mirror script (R. Tankle \& $\mathrm{K}$. Heilman, 1983, Brain and Language, $19,115-123)$. We tested this claim by comparing the mirror writing ability of normal right- and left-handed adults as a function of hand used and hand use order. Our results do not support the notion of a left-hander superiority in mirror writing since, in two different experiments, right-handed individuals were found to be as good as left-handers. Instead, we obtained evidence for an order effect such that in both experiments, mirror writing was faster in those who started the task with their right hand. (-) 1989 Academic Press, Inc.
\end{abstract}

Spontaneous occurrences of mirror writing are often observed among young children (Cornell, 1985; Orton, 1937), in adults following left cerebral hemisphere brain injury (Heilman, Howell, Valenstein, \& Rothi, 1980; Schott, 1980; Streifler \& Hofman, 1976; Woods \& Teuber, 1978) and in brain-intact left-handers (Jung, 1981). Leonardo da Vinci, a lefthander, wrote in mirror script. Indeed, clinical reports of spontaneous mirror writing in left-handed individuals, including those who were forced

Portions of this research were presented at the annual meeting of the International Neuropsychological Society in San Diego, February 1985. The research was supported by the John D. and Catherine T. MacArthur Foundation Transition Research Network at the Univcrsity of California, San Diego. Preparation of this manuscript was supported by a Summer Research Grant awarded to the first author by the College of Liberal Arts, Texas A\&M University. We thank Gay Miyamoto and Karen Hampton for running the experiments and Tom Battocletti for assistance in data analysis. Michael Peters provided useful references and Harry A. Whitaker drew our attention to the work of Joseph Elder. We are also grateful to two anonymous reviewers for their comments on a previous draft. Requests for reprints should be sent to the first author c/o Department of Psychology, Texas A \& M University, College Slation, TX 77843-4235. 
to write with their right hand, date back to the late 19th century (Clapham, 1895; Carmichael \& Cashman, 1932; Critchley, 1928; Fuller, 1916; Ireland, 1881; Mills, 1894; Rudolph, 1903; Russell, 1900; Sweeney, 1900; see Corballis \& Beale, 1976, and Harris, 1980, for further discussion).

While the inclination of sinistrals to engage in spontaneous mirror writing has been well documented, less attention has been paid to actual performance differences between left- and right-handers in mirror writing. The earliest empirical study of mirror writing in normal adults was conducted some 50 years ago (see Elder, 1927, and Whitaker, 1987), but studies comparing right- and left-handers' ability to mirror write are fairly recent (e.g., Parlow \& Kinsbourne, 1983). A direct comparison of rightand left-handers was undertaken by Tankle and Heilman $(1982,1983)$ who reported faster mirror writing and better mirror reading performance among normal left- than right-handers. Subsequent studies, however, have failed to replicate the handedness effect in mirror reading (Bradshaw, Nettleton, Wilson, \& Burden, 1985; Bradshaw \& Bradshaw, 1988) and considerable variability among left-handers has been observed in the execution of mirror script (Peters, 1983). The present study sought to investigate the claim by Tankle and Heilman (1983) of a left-hander advantage in mirror writing ability.

\section{Tankle and Heilman (1983)}

Tankle and Heilman (1983) examined mirror writing ability in two experiments. In the first experiment they found that left-handed adults were faster than right-handers at writing in mirror script using either their preferred or nonpreferred hand. They also reported a practice effect in mirror writing speed, but it was significant in right-handers only. An analysis of errors made while mirror writing indicated a superiority on the part of left-handers, but only when the preferred hand was used. When the nonpreferred hand was used, left-handers made more errors than right-handers, suggesting the existence of a speed-accuracy tradeoff.

In their second experiment, Tankle and Heilman (1983) compared speed of normal and mirror writing with the preferred hand in right- and left-handers. No group differences were found in normal writing speed. For mirror writing, the left-hander advantage found in their first experiment was again obtained.

Methodological issues. Two aspects of the Tankle and Heilman (1983) study restrict the interpretation of their results.

The first concerns the manner in which the researchers chose to analyze their data. From how the results are presented, it would appear that several one-way analyses of variance were used to assess the contribution of the various factors of interest (e.g., hand used, order of hand used, and hand dominance). Given the problems associated with con- 
ducting multiple analyses of the same data set (i.e., an increased likelihood of a Type I error), a more judicious approach would have been to incorporate all of the factors into a single analysis of variance. A multifactorial design not only yields a more reliable measure of main effects but also allows for interaction effects to be assessed.

The second problem has to do with the specification of subjects' handedness. Subjects were classified as right- or left-handed solely on the basis of self-report of hand used to write. No additional information was solicited in terms of degree of hand dominance, a history of forced switch in hand usage, etc. Also lacking was information about the incidence of left-handedness in the subjects' immediate family. Given the increasing recognition of the multitude of dimensions along which hand dominance may be classified, the insensitivity to such variation in the subject selection criteria used by Tankle and Heilman makes it difficult to assess the generalizability of their findings.

The present experiments on normal and mirror writing ability in rightand left-handers were designed with the above methodological considerations in mind.

\section{EXPERIMENT 1}

The first experiment sought to replicate the Tankle and Heilman (1983) finding of a left-hander advantage in mirror writing speed and accuracy.

\section{Method}

Subjects. A total of 32 college students from a larger sample who responded to notices calling for right- or left-handed people participated in the experiment. There were 16 righthanders (mean age 20.2 years) and 16 left-handers (mean age 20.6 years), including an equal number of men and women per group. Subjects were classified as right- or lefthanded on the basis of their responses on a 10-item adaptation of the Edinburgh Handedness Inventory (Oldfield, 1971). The criterion for inclusion in the study was reported use of one hand for 7 or more items on the inventory. The incidence of familial left-handedness was $18.8 \%$ in the right-handers and $43.8 \%$ in the left-handers. Although it was not possible to equate subjects on the basis of their hand posture while writing, this characteristic was coded in our sample so that its potential influence on the speed or quality of pen movement in the nonpreferred direction may be systematically investigated in future work. With the exception of one subject, all of the right-handers reported using a noninverted hand posture for normal writing; one female reported using an inverted hand posture. Among the lefthanders, 6 of the males (75\%) reported using an inverted hand posture compared to 1 of the females $(12.5 \%)$. None of the subjects had been forced to change their handedness in early childhood.

Stimuli and procedure. A list of nine sentences was used. With minor modifications, e.g., substitution of "University of California" for "University of Florida," six of the sentences were the same as those used by Tankle and Heilman (1983) and three were new sentences. The list was in turn subdivided into three subsets each containing three sentences. ' One subset (the new sentences) was assigned to the normal writing condition, the other two to the mirror writing condition.

\footnotetext{
${ }^{1}$ A list of the stimuli is available upon request.
} 
For the mirror condition, subjects were told that they would be timed on their ability to write a set of sentences in lower case, cursive, mirror-reversed script. They were given a mirror to view their output while practicing writing in mirror script. After it was clear that they understood the task, the experiment was initiated. Each of the sets of three sentences was read aloud once to the subjects, who were to listen to the complete sentence before beginning the task of mirror writing. The examiner, who was blind to the hypothesis underlying the experiment, recorded subjects' writing speed for each sentence, using a stopwatch accurate to the nearest hundredth of a second.

Half of the right-handers and half of the left-handers used their right hand to write the first set of three sentences (Block 1) followed by their left hand to write the second set of sentences (Blockk 2); the remainder were tested with their left hand first in Block 1, followed by their right hand in Block 2. The particular sentence sets assigned to a given experimental condition were also counterbalanced across subjects.

The third set of sentences comprised the normal writing condition. In this condition, subjects were tested on their speed of writing the sentences with their preferred hand. This condition was administered after the two mirror writing conditions were completed.

Results

Normal writing speed. An analysis of the normal writing scores indicated no significant main or interaction effects of group or sex in speed of writing with the preferred hand.

Mirror writing speed. Mirror writing response latencies per subject were averaged across each of the sets of three stimulus sentences to produce a mean reaction time score for each hand. A preliminary analysis of variance indicated no significant main effect or interaction effects of sex. The data were thus collapsed on this factor and analyzed in a threeway factorial design as a function of Group (right- vs. left-handers), Hand Order (right hand used first or second), and Trial block (first vs. second). It should be remembered that the hand used to mirror write in Block 1 was different from the hand used in Block 2.

Table 1 summarizes the mean performance of right- and left-handers as a function of Hand Order and Block. There was a significant main effect of Block $[F(1,28)=50.44, p<.00001]$ indicating that subjects improved in their mirror writing speed from the first to the second block (81.53 vs. $65.06 \mathrm{sec}$, respectively). In addition, there was a significant main effect of Hand Order $[F(1,28)=4.64, p<.04]$. This effect revealed

TABLE 1

Experiment 1: Mean Mirror Writing Times (IN SEC)

\begin{tabular}{lcccc}
\hline Group & \multicolumn{2}{c}{ Right hand } & \multicolumn{2}{c}{ Left hand } \\
\hline I. Right hand first & Mean & $(S D)$ & Mean & $(S D)$ \\
$\quad$ Left-handers & 73.0 & $(24.4)$ & 53.5 & $(16.4)$ \\
$\quad$ Right-handers & 75.8 & $(15.1)$ & 63.9 & $(13.8)$ \\
II. Left hand first & & & & \\
$\quad$ Left-handers & 70.1 & $(15.5)$ & 85.4 & $(18.4)$ \\
Right-handers & 72.8 & $(19.5)$ & 92.0 & $(25.1)$ \\
\hline
\end{tabular}


that, for right- and left-handers alike, performance on the mirror writing task was faster when the right hand was used first than when it was used second ( 66.53 vs. $80.06 \mathrm{sec}$, respectively). Whereas Tankle and Heilman (1983) reported that left-handers were significantly faster than right-handers in both the preferred-hand-first and second conditions, the present analysis found no main or interaction effects involving the group variable. The mean overall performance of right-handers $(76.1 \mathrm{sec})$ was not significantly different from that of left-handers $(70.5 \mathrm{sec}),[F(1,18)=0.79$, $p=0.38]$.

Mirror writing accuracy. The mean number of letters per sentence set not correctly mirror-reversed was entered into a $2 \times 2 \times 2 \times 2$ analysis of variance (Group by Sex by Hand Order by Block). Table 2 provides a summary of the error rates.

There were no main effects and two significant interaction effects: Sex $\times$ Hand Order $[F(1,24)=6.39, p<.02]$ and Group $\times$ Block $\times$ Hand Order $[F(1,24)=6.17, p<.02]$. A post hoc test of the Sex by Hand Order interaction using Tukey's hsd statistic revealed that women made fewer errors than men, especially in the left-hand-first condition ( $1.2 \mathrm{vs.}$ 3.3 , respectively), and that males made fewer errors in the right-handfirst versus the left-hand-first condition (1.2 vs. 3.3, respectively); Tukey's $h s d=2.1, d f=24, p<.05$.

A post hoc test of the three-way interaction revealed that right-handed subjects in particular made fewer errors in the right-hand-first as compared to the left-hand-first condition, Tukey's $h s d=0.9, d f=24, p<$ .05 .

\section{Discussion}

One finding from this experiment is that mirror writing speed improves with (opposite hand) practice: performance was about $16 \mathrm{sec}$ faster in the second block than in the first. The fact that this was the case regardless of subjects' hand preference conflicts with the finding in Tankle and Heilman (1983) in which only right-handers benefited from practice.

A second and more interesting outcome of the present study is that

TABLE 2

Experiment 1: Mean Mirror Writing Error Rates

\begin{tabular}{lcccc}
\hline Group & \multicolumn{2}{c}{ Right hand } & \multicolumn{2}{c}{ Left hand } \\
\hline I. Right hand first & Mean & $(S D)$ & Mean & $(S D)$ \\
$\quad$ Left-handers & 1.8 & $(1.3)$ & 2.0 & $(1.5)$ \\
$\quad$ Right-handers & 1.4 & $(1.7)$ & 1.1 & $(0.8)$ \\
II. Left hand first & & & & \\
$\quad$ Left-handers & 1.6 & $(1.2)$ & 2.5 & $(1.5)$ \\
Right-handers & 2.8 & $(1.8)$ & 1.7 & $(1.2)$ \\
\hline
\end{tabular}


mirror writing performance is apparently facilitated by initial use of the right hand (or, alternatively, that it is inhibited by initial use of the left hand). Subjects who began the task with their right hand were on average $14 \mathrm{sec}$ faster than those who began with their left hand. Since this finding was true of right- and left-handers, it goes against Tankle and Heilman's report of a general left-hander superiority in mirror writing speed. Indeed, the present study found no significant handedness difference in mirror writing speed nor did this variable interact with any of the other variables. The error analysis indicates that a speed-accuracy trade-off was unlikely, for subjects tended to make fewer errors in the right-hand-first condition.

The apparent facilitatory effect of the right hand needs to be further explored. Given that the design of the experiment was such that the second block of trials involved a change in the hand used to mirror write, it is not clear to what extent the improved performance reflects a facilitation effect of right-hand use or of practice per se. To determine if there is something uniquely facilatatory about prior practice with the right hand (or inhibitory about prior left-hand practice) apart from samehand practice per se, a second experiment was conducted.

\section{EXPERIMENT 2}

In this experiment a total of three blocks of mirror-writing trials were administered. The first two blocks involved the same hand to mirror write while the third block involved a change in the hand used. Thus, half of the right- and left-handers received two blocks of right-handed mirror writing followed by one block of left-handed mirror writing. The remaining subjects began with two blocks of left-handed mirror writing. As in Experiment 1, normal writing speed of right- and left-handers was compared. However, in this case, normal writing performance was assessed for each hand (preferred hand and nonpreferred hand).

\section{Method}

Subjects. A total of 36 college students were tested. These included 18 right- and 18 left-handers, screened on the basis of the Edinburgh Handedness Inventory (Oldfield, 1971), using the same criteria as in the previous experiment. There were an equal number of men and women per group. The mean age of right-handers was 20.5 years and of left-handers 23.2 years. Subjects in this experiment had not participated in Experiment 1.

All but one right-hander reported a noninverted hand posture when writing; the exception was a male right-hander who used an inverted hand posture. Eight out of the nine lefthanded females $(89 \%)$ reported using a noninverted hand posture when writing normally; the remainder reported using an inverted posture. Three of the nine left-handed males (33\%) reported using a noninverted posture and the remainder reported using an inverted hand posture. Eight of the left-handers (44.4\%) had family members who were left-handed; eight right-handers also had left-handed family members. None of the subjects had been forced to change their hand usage.

Stimuli and procedure. Fifteen sentences were constructed and randomly assigned to five blocks of three sentences each, containing an equal number of total syllables. Two of the blocks were assigned to the normal writing conditions and three blocks involved 
mirror writing. Normal writing conditions preceded and followed the mirror writing conditions. Order of hand used was counterbalanced across subjects such that half of the subjects per handedness group began with their right hand and ended with the left hand and the remainder were assigned the converse order.

In the first mirror writing block, subjects began with the same hand used for the initial normal writing condition that preceded mirror writing. They continued using that hand in the second block of mirror writing and then switched to their other hand in the third block. The session concluded with a normal writing condition using the same hand as was used in Block 3 of the mirror writing condition. The experimental design is diagrammed in Fig. 1.

Data analysis. Normal writing latencies and mirror writing scores were analyzed separately. In addition, two separate analyses of mirror writing latencies were undertaken. The first considered the effects of same-hand practice. The second analysis was performed on the averaged mirror writing data from the same-hand blocks (Blocks 1 and 2) and the mirror writing scores from Block 3. This second analysis allows a direct comparison with that of Experiment 1.

\section{Results}

Normal writing speed. The normal writing data were entered into a four-way analysis of variance as a function of Group (right- vs. lefthanders), Sex, Hand Order (right hand first vs. second), and Block (first vs. second). Since the variable of Sex did not emerge as a significant effect, it was removed and the data were reanalyzed.

The analysis indicated a main effect of Block, $[F(1,32)=11.30, p<$

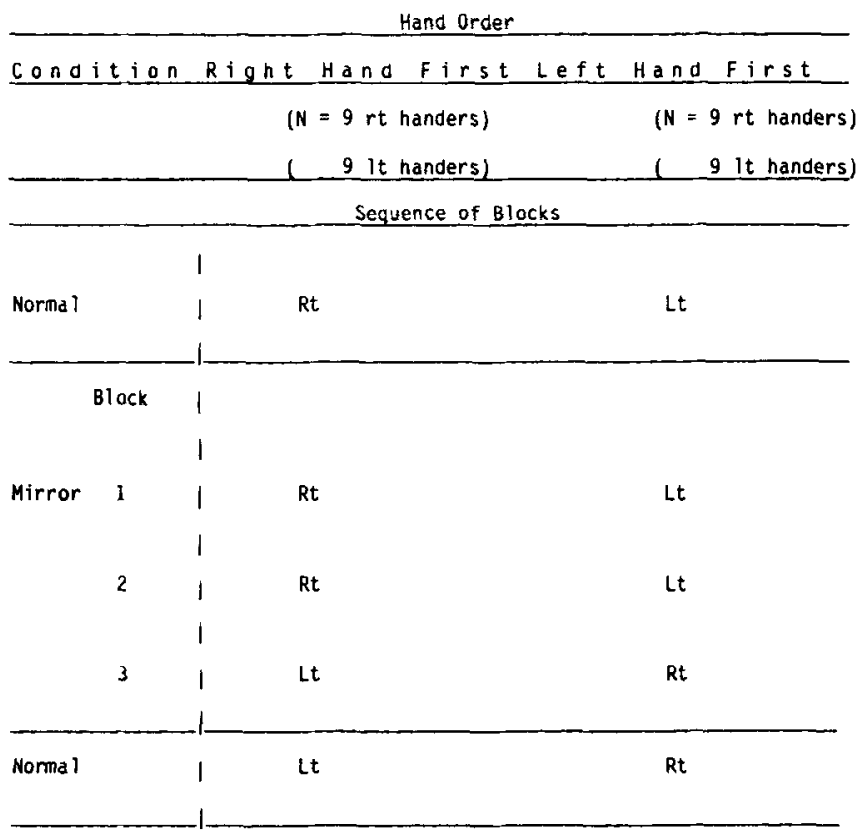

Fig. 1. A depiction of the design of Experiment 2. 
$.002]$ and of Group, $[F(1,32)=5.65, p<.02]$. In addition, there were two interaction effects: Block by Hand Order $[F(1,32)=10.37, p<$ $.003]$ and Group by Block by Hand Order $;[F(1,32)=445.31, p<$ $.00001]$. The three-way interaction is presented in Table 3 .

The interaction revealed that performance is fastest when subjects use their preferred hand to write, regardless of whether the preferred hand is used first or second, Tukey's $h s d=6.0, d f=32, p<.05$.

Mirror writing speed (Blocks 1, 2, and 3). A preliminary analysis revealed no significant main or interaction effect of sex. This variable was therefore removed and the data were reanalyzed as a function of Group (right- vs. left-hander), Hand Order (right hand used first vs. second), and Testing Block (three in all). Table 4 presents a summary of the mean values by group. The analysis revealed a main effect of Block, $[F(2,64)$ $=27.47, p<.00001]$. Subjects were faster in Block $2(65.1)$ than in Block 1 (76.6), and in Block 3 (66.6) than in Block $1(p<.05)$, whereas no significant difference was found between Blocks 2 and 3, Tukey's $h s d=5.5, d f-64, p<.05$.

A main effect of Hand $\operatorname{Order}[F(1,32)=7.85, p<.008]$ was also obtained. This indicated that responses were faster when the right hand was used first $(63.0 \mathrm{sec})$ than when it was used second $(75.8 \mathrm{sec})$. As in the previous experiment, the main effect of Group was not significant, $[F(1,31)=0.56, p=.46]$, nor did this variable interact with any other variable.

Mirror writing speed (Blocks 1 and 2 averaged, vs. 3). When Block 1 and 2 scores were averaged and compared with those of Block 3 in a three-way analysis of variance by Group, Hand Order, and Block a significant effect of Hand Order was obtained, $[F(1,32)=7.40, p<$ $.01]$. As in Experiment 1 subjects in the right-hand-first condition mirror wrote faster than those in the right-hand-second condition. In addition, a main effect of Block was obtained $[F(1,32)=6.80, p<.01]$, which indicated faster responses in the second block. See Table 5. There were no other significant effects.

TABLE 3

Experiment 2: Mean Performance (in sec) on Normal Writing Task as a Function of Group, Hand Order, and Block

\begin{tabular}{lcccr}
\hline Group & \multicolumn{2}{c}{ Right hand } & \multicolumn{2}{c}{ Left hand } \\
\hline I. Right hand first & Mean & $(S D)$ & Mean & $(S D)$ \\
$\quad$ Left-handers & 32.9 & $(5.8)$ & 17.2 & $(3.9)$ \\
$\quad$ Right-handers & 15.9 & $(2.0)$ & 45.0 & $(6.8)$ \\
II. Left hand first & & & & \\
$\quad$ Left-handers & 37.9 & $(6.4)$ & 17.0 & $(1.9)$ \\
Right-handers & 18.3 & $(3.8)$ & 38.9 & $(7.1)$ \\
\hline
\end{tabular}


TABLE 4

Experiment 2: Mean Performance (In Sec) on Mirror Writing Task as a Function of Grour, Hand Order, and Block

\begin{tabular}{lccccccc}
\hline Group & \multicolumn{2}{c}{ Block 1 } & \multicolumn{2}{c}{ Block 2 } & \multicolumn{2}{c}{ Block 3 } \\
\hline I. Right hand first & Mean & $(S D)$ & Mean & $(S D)$ & Mean & $(S D)$ \\
$\quad$ Left-handers & 64.1 & $(11.4)$ & 54.4 & $(11.3)$ & 55.0 & $(15.5)$ \\
$\quad$ Right-handers & 73.0 & $(13.4)$ & 63.3 & $(10.1)$ & 68.3 & $(12.2)$ \\
$\begin{array}{l}\text { II. Left hand first } \\
\quad \text { Left-handers }\end{array}$ & 87.9 & $(16.3)$ & 74.0 & $(15.0)$ & 70.9 & $(16.5)$ \\
$\quad$ Right-handers & 81.2 & $(18.8)$ & 68.6 & $(17.6)$ & 72.3 & $(17.2)$ \\
\hline
\end{tabular}

Mirror writing accuracy (Blocks 1 and 2 averaged, vs. 3). The mean number of incorrectly reversed letters per stimulus set were analyzed as a function of Sex, Group, Hand Order, and Block (Blocks 1 and 2 averaged, vs. Block 3). The analysis indicated a significant Group $\times$ Order interaction, $[F(1,28)=14.35, p<.0007]$. See Table 6 for a summary of the error rates.

Breakdown of the analysis revealed that left-handers made more errors than right-handers in the left-hand-first condition, Tukey's $h s d=2.7$, $d f=28, p<.05$, and made fewer errors in the right-hand-first than in the left-hand-first condition.

\section{GENERAL DISCUSSION}

Findings from our two experiments challenge the claim by Tankle and Heilman (1983) that left-handers are generally better than right-handers at writing in mirror script. In neither experiment was evidence obtained of handedness-based differences in mirror writing speed, let alone of a left-hander superiority. Another discrepancy between the present study and that of Tankle and Heilman lies in the influence of practice; in the latter study only right-handers appeared to benefit from prior practice with the other hand. By contrast, in both experiments of the present

TABLE 5

Experiment 2: Mean Mirror Writing Times (IN SEC) for Blocks 1 and 2 vs. 3

\begin{tabular}{lcccc}
\hline Group & \multicolumn{2}{c}{ Right hand } & \multicolumn{2}{c}{ Left hand } \\
\hline I. Right hand first & Mean & $(S D)$ & Mean & $(S D)$ \\
Left-handers & 59.2 & $(11.4)$ & 55.0 & $(15.5)$ \\
$\quad$ Right-handers & 68.2 & $(11.8)$ & 68.3 & $(12.2)$ \\
II. Left hand first & & & & \\
$\quad$ Left-handers & 70.9 & $(16.5)$ & 80.9 & $(15.6)$ \\
Right-handers & 72.3 & $(17.2)$ & 74.9 & $(18.2)$ \\
\hline
\end{tabular}


TABLE 6

Experiment 2: Mean Mirror Writing Error Rates

\begin{tabular}{lcccc}
\hline Group & \multicolumn{2}{c}{ Right hand } & \multicolumn{2}{c}{ Left hand } \\
\hline I. Right hand first & Mean & $(S D)$ & Mean & $(S D)$ \\
$\quad$ Left-handers & 3.6 & $(1.9)$ & 3.4 & $(1.8)$ \\
$\quad$ Right-handers & 5.3 & $(2.1)$ & 5.6 & $(2.5)$ \\
II. Left hand first & & & & \\
$\quad$ Left-handers & 6.2 & $(2.1)$ & 7.4 & $(2.6)$ \\
Right-handers & 4.3 & $(2.2)$ & 4.0 & $(1.3)$ \\
\hline
\end{tabular}

study, right- and left-handers showed a significant improvement in performance across blocks of trials.

Finally, we observed an apparent facilitation with prior experience with the right hand. See Fig. 2. The fact that this effect was replicated in Experiment 2, using a different set of subjects, suggests that it is not a sampling artifacl, and the error analysis reveals that there was no speed-accuracy trade-off operating.

What might this right-hand-first facilitation of mirror writing speed reflect? It may be that the motor program set into motion by practice with a particular hand makes it easier for the other hand to execute the task (see Milisen \& Van Riper, 1939, for a similar effect on a rotary task). However, this suggestion does not account for the observed asymmetry observed, namely that initial use of the right hand facilitated performance more than initial use of the left hand. Perhaps a biomechanical effect best explains the results. According to Brown, Knauft, and Rosenbaum (1948), movements made away from the body are executed more accurately than movements directed toward the body. If we can extend this principle to include speed of movement, then we would expect both right- and left-handers to perform faster when writing with their left hand since, in this case, the direction of mirror writing would be

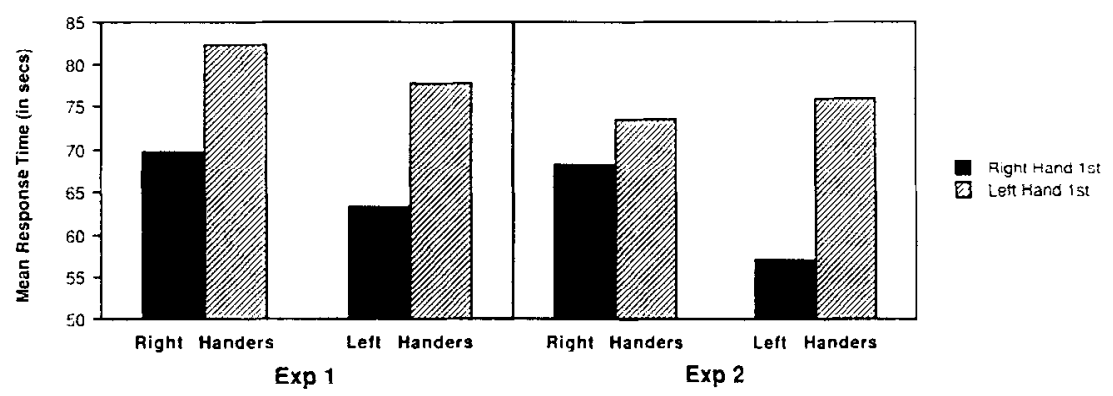

FIG. 2. A summary of mirror writing speed in Experiments 1 and 2 as a function of Hand Order. 
away from the body. This effect might appear enhanced when the lefthand mirror writing condition is preceded by a right-handed one. Thus, the apparent facilitation observed in our results from prior use of the right hand could reflect a disinhibiting effect of movements made toward the body on subsequent movements made away from the body. This explanation accords with one of the three hypotheses discussed by Tankle and Heilman (1983) and posits hand-based rather than handedness-based differences in mirror writing skill. However, this explanation does not fully account for our results since there was no interaction of Hand Order by Block. That is, initial use of the right hand improved performance equally in the first block (right-hand use) as in the second block (lefthand use).

Whatever the mechanism underlying the observed asymmetry, it is clear from the present study that mirror writing ability does not come more easily to a particular handedness group (i.e., left-handers) but rather that both right- and left-handed individuals benefit equally from practice in mirror writing. Moreover, mirror writing appears to be particularly facilitated in both handedness groups when the task is initiated by the right hand. It is this finding, we suggest, that is of theoretical interest and in need of further investigation.

\section{REFERENCES}

Bradshaw, J. L., \& Bradshaw, J. A. 1988. Reading mirror-reversed text: Sinistrals really are inferior. Brain and Language, 33, 189-192.

Bradshaw, J., Nettleton, N., Wilson, L., \& Burden, V. 1985. Mirror reading in right and lcft handers: Are sinistrals really superior? Brain and Language, 26, 322-331.

Brown, J., Knauft, E., \& Rosenbaum, G. 1948. The accuracy of positioning movements as a function of their direction and extent. American Journal of Psychology, 61, 167182.

Carmichael, L., \& Cashman, H. 1932. A study of mirror writing in relation to handedness and perceptual and motor habits. Journal of General Psychology, 6, 296-329.

Clapham, A. 1985. A case of mirror writing. Quarterly Medical Journal, 3, 333-335.

Corballis, M., \& Beale, I. 1976. The psychology of left and right. Hillsdale: Lawrence Erlbaum.

Cornell, J. 1985. Spontaneous mirror writing in children. Canadian Journal of Psychology, 39(1), 174-179.

Critchley, M. 1928. Mirror-writing. London: Kegan Paul, Trench, Trübner.

Elder, W. 1927. Studies in psychology. London: Heinemann.

Fuller, J. 1916. The psychology and physiology of mirror writing (Vol. 2, pp. 199-205). University of California Publications in Psychology.

Harris, L. 1980. Left-handedness: Early theories, facts, and fancies. In J. Herron (Ed.), Neuropsychology of left-handedness. New York: Academic Press.

Heilman, K., Howell, G., Valenstein, F., \& Rothi, I. 1980. Mirror-reading and writing in association with right-left spatial disorientation. Journal of Neurology, Neurosurgery, and Psychiatry, 43, 774-780.

Ireland, W. 1881. On mirror-writing and its relation to left-handedness and cerebral disease. Brain, 4, 361-367.

Jung, R. 1981. Perception and action. In J. Szentagothai, M. Palkovitz, \& J. Hamori (Eds.), 
Regulatory functions of the CNS, principles of motion and organization. Oxford; Pergamon.

Milisen, R., \& Van Riper, C. 1939. Differential transfer of training in a rotary activity. Journal of Experimental Psychology, 24, 640-646.

Mills, C. 1894. Mirror writing. Journal of Mental Disorders, 21, 85-91.

Oldfield, R. C. 1971. The assessment and analysis of handedness: The Edinburgh inventory. Neuropsychologia, 9, 97-113.

Orton, S. 1937. Reading, writing, and speech problems in children. New York: Norton.

Parlow, S., \& Kinsbourne, M. 1983, February. Bilateral transfer of training: Constraints within the intact brain? Paper presented at annual meeting of the International Neuropsychological Society, Mexico City.

Peters, M. 1983. Inverted and uninverted lefthanders compared on the basis of motor performance and measures related to the act of writing. Australian Journal of Psychology, 35(3), 405-416.

Rudolph, R. 1903. Mirror-writing. Canadian Practical Review, 28, 83-88.

Russell, J. 1900. A case of mirror writing. Birmingham Medical Review, 68, 95-101.

Schott, G. D. 1980. Mirror movements of the left arm following peripheral damage to the preferred right arm. Journal of Neurology, Neurosurgery, and Psychiatry, 43, 768773.

Streifler, M., \& Hofman, S. 1976. Sinistrad mirror writing and reading after brain concussion in a bi-systemic (oriento-occidental) polyglot. Cortex, 12, 356-364.

Sweeney, A. 1900. Mirror-writing, inverted vision, and allied ocular defects. Saint Paul Medical Journal, 2, 374-384.

Tankle, R., \& Heilman, K. 1982. Mirror reading in right and left handers. Brain and Language, 17, 124-132.

Tankle, R., \& Heilman, K. 1983. Mirror writing in right handers and left handers. Brain and Language, 19, 115-123.

Whitaker, H. 1987, March. Joseph Elder. Paper presented at annual meeting of the Body for the Advancement of Brain, Behavior, and Language Enterprises, Niagara Falls, Ontario.

Woods, B., \& Teuber, H. 1978. Mirror movements after childhood hemiparesis. Neurology, 28, 1152-1158. 